\title{
Technè
}

La science au service de l'histoire de l'art et de la préservation des biens culturels

45 | 2017

Bronzes grecs et romains : études récentes sur la statuaire antique

\section{Pour une meilleure compréhension du procédé de soudage de la grande statuaire antique en bronze : analyses et modélisation expérimentale}

Towards a better understanding of the welding process used in Greek and Roman large bronze statuary: analyses and experimental modelling

Aurélia Azéma, Daniel Chauveau, Gaëlle Porot, Florent Angelini et Benoît Mille

\section{OpenEdition Journals}

Édition électronique

URL : http://journals.openedition.org/techne/1286

DOI : $10.4000 /$ techne.1286

ISSN : 2534-5168

Éditeur

C2RMF

Édition imprimée

Date de publication : 1 mai 2017

Pagination : 73-83

ISBN : 978-2-7118-6408-9

ISSN : $1254-7867$

Référence électronique

Aurélia Azéma, Daniel Chauveau, Gaëlle Porot, Florent Angelini et Benoît Mille, « Pour une meilleure compréhension du procédé de soudage de la grande statuaire antique en bronze : analyses et modélisation expérimentale», Technè [En ligne], 45 | 2017, mis en ligne le 19 décembre 2019, consulté le 25 juillet 2020. URL : http://journals.openedition.org/techne/1286 ; DOI : https://doi.org/10.4000/ techne. 1286

La revue Technè. La science au service de l'histoire de l'art et de la préservation des biens culturels est mise à disposition selon les termes de la Licence Creative Commons Attribution - Pas d'Utilisation Commerciale - Pas de Modification 4.0 International. 
Aurélia Azéma

Daniel Chauveau

Gaëlle Porot

Florent Angelini

Benoît Mille

\section{Pour une meilleure compréhension du procédé de soudage de la grande statuaire antique en bronze : analyses et modélisation expérimentale}

Towards a better understanding of the welding process used in Greek and Roman large bronze statuary: analyses and experimental modelling
Résumé. Cet article présente l'avancement de nos recherches consacrées à l'étude technologique et à la modélisation expérimentale des assemblages soudés de la grande statuaire antique en bronze. La méthode adoptée et les résultats obtenus précisent le mécanisme de liaison, et conduisent à proposer une nouvelle dénomination du procédé : soudage par fusion au bronze liquide. Nous mettons aussi en évidence l'importance de la préparation au soudage, et une typologie de la forme des joints soudés est ébauchée. Nous présentons enfin les derniers développements des examens par ultrasons qui offrent à terme la perspective de tomographies à l'aide d'instruments portables sur le lieu même de conservation des cuvres.

Mots-clés. Sculpture, bronze, Antiquité, soudage par fusion, contrôles non destructifs.

\begin{abstract}
This paper summarizes the progress made in our research on the technological study and experimental modelling of welded assemblages used in Greek and Roman large bronze statuary. The chosen method and results obtained clarify the binding mechanism and lead us to propose a new name for the process: flow fusion welding. We also demonstrate the importance of the preparation stage in welding and outline a typology of the shape of the welded joins. Lastly, we present the latest developments in ultrasonic testing, which, in the long run, envisage tomography with portable instruments being used in the actual place where works are held.

Keywords. Sculpture, bronze, Antiquity, fusion welding, non-destructive testing.
\end{abstract}

\section{Introduction}

Dès le XIX ${ }^{\mathrm{e}}$ siècle, quelques observateurs clairvoyants avaient envisagé la possibilité que les grands bronzes antiques n'aient pas été coulés en un seul jet, mais fabriqués en pièces séparées assemblées par un procédé très efficace de soudage ${ }^{1}$. Les recherches du $\mathrm{XX}^{\mathrm{e}}$ siècle se sont en priorité focalisées sur l'étude technologique du moulage (procédé indirect de fonte à la cire perdue $)^{2}$ et sur l'analyse chimique du bronze (éléments d'alliage et éléments en traces) ${ }^{3}$. L'étude des assemblages n'a vraiment vu le jour qu'au début des années 1970 avec les recherches pionnières de Steinberg ${ }^{4}$, confirmées dans les années 1980-1990 par les résultats obtenus sur les guerriers de Riace $^{5}$, le dépôt de bronzes d'Augst ${ }^{6}$ et les grands bronzes des collections nord-américaines ${ }^{7}$.

Il a cependant fallu attendre le lancement d'un programme étudiant l'évolution des techniques de la grande statuaire métallique antique, piloté par le Centre de Recherche et de Restauration des Musées de France et le département des Antiquités grecques, étrusques et romaines du musée du
Louvre, pour prendre la pleine mesure de l'importance des techniques de soudage dans la fabrication des grands bronzes. Avec l'étude du cheval de Neuvy-en-Sullias, il a par exemple été montré que la statue a été coulée en 20 pièces séparées puis soudées ; mises bout à bout, les soudures formeraient un cordon de plus de cinq mètres de longueur ${ }^{8}$. Pour les grands dauphins de Vienne, certaines soudures faites d'un seul tenant mesurent près d'un mètre de longueur ${ }^{9}$ ! Ce faisant, nous avons montré qu'il existe une grande variabilité dans la technique antique de soudage, pour la préparation du joint soudé comme pour la mise en œuvre de l'assemblage, faisant du procédé de soudage un marqueur chronologique et/ou régional de première importance. Une forme particulière de soudure dite en "plate-forme " a ainsi récemment été mise en évidence ${ }^{10}$. Cette modalité d'assemblage n'existe pas avant la période hellénistique, ce qui constitue un argument important pour ne pas attribuer l'Apoxyomène de Croatie au IV siècle avant J.-C., ou l'Apollon de Piombino au V $V^{\mathrm{e}}$ siècle avant J.-C. ${ }^{11}$ Une autre forme de soudure dite " en cuvettes » (voir ci-après) semble avoir joué une importance particulière

Aurélia Azéma, ingénieur d'étude, LRMH, CRC, UMR 3224 (aurelia.azema@culture.gouv.fr). Daniel Chauveau, directeur Innovation, expert senior en contrôle non destructif, Institut de Soudure Groupe, direction Innovation, Paris Nord 2 (d.chauveau@isgroupe.com).

Gaëlle Porot, responsable de projet CND de R\&D, Institut de Soudure Groupe, Plateforme RDICND Yutz (g.framezelle@isgroupe.com).

Florent Angelini, Institut de Soudure Groupe, IS Experts, Paris Nord 2 (florent.angelini@vale.com). Benoît Mille, ingénieur d'étude, C2RMF et Préhistoire et Technologie, UMR 7055, Nanterre (benoit.mille@culture.gouv.fr). 


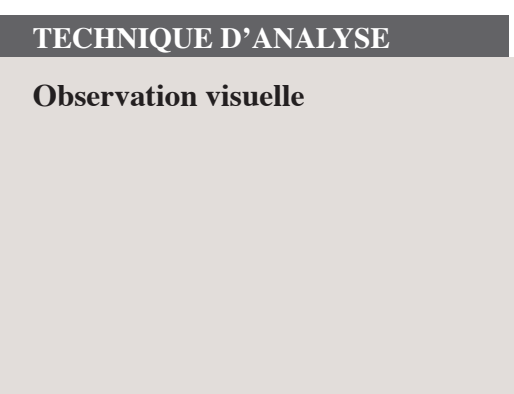

Radiographie

\section{EXEMPLE D'APPLICATION}
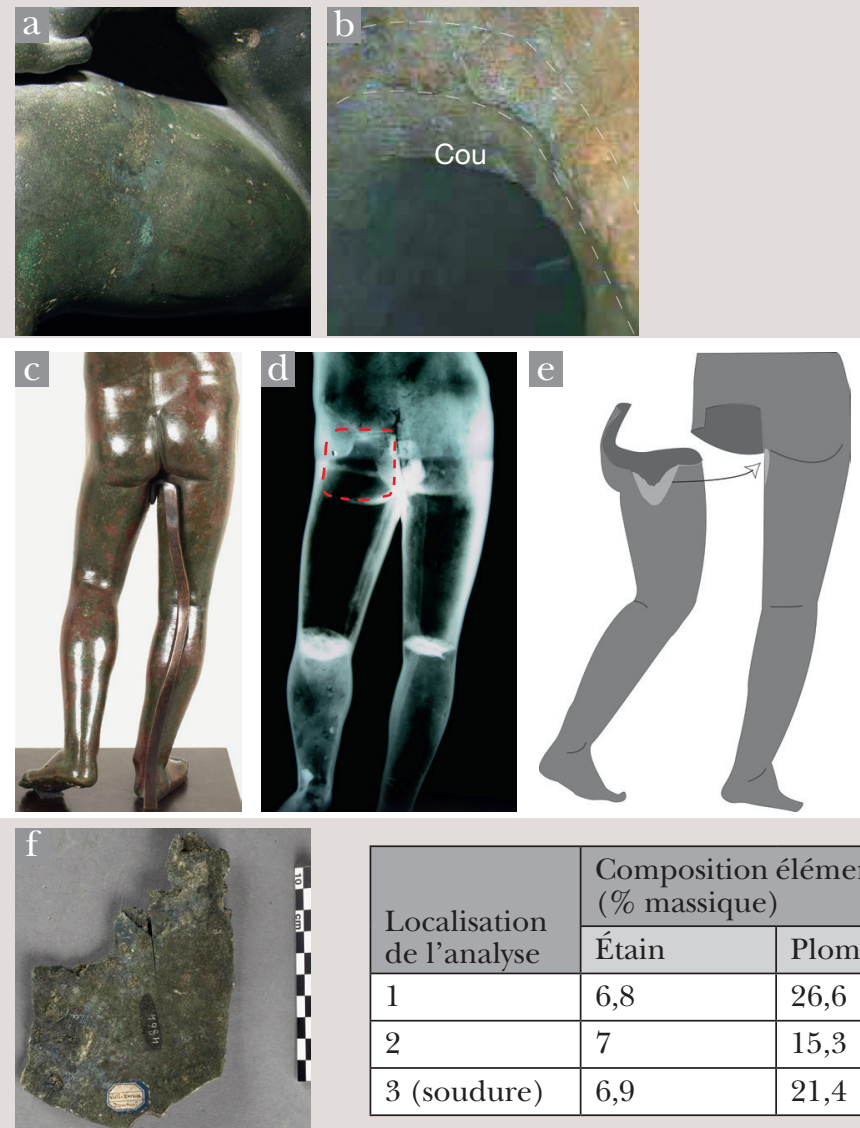

\begin{tabular}{|l|l|l|l|}
\hline \multirow{2}{*}{$\begin{array}{l}\text { Localisation } \\
\text { de l'analyse }\end{array}$} & \multicolumn{2}{|l|}{$\begin{array}{l}\text { Composition élémentaire } \\
\text { \% massique) }\end{array}$} & $\begin{array}{l}\text { Température } \\
\text { de complète } \\
\text { fusion }\left({ }^{\circ} \mathrm{C}\right)\end{array}$ \\
\cline { 2 - 4 } 1 & Étain & Plomb & 900 \\
\hline 2 & 6,8 & 26,6 & 965 \\
\hline 3 (soudure) & 6,9 & 15,3 & 960 \\
\hline
\end{tabular}

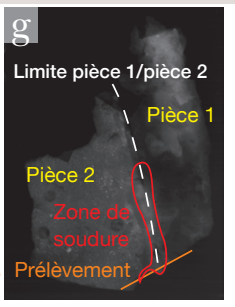

Zones caractéristiques du joint soudé par fusion

Zone fondue : zone où, durant le processus de soudage, l'état liquide a été obtenu.

Zone de liaison : frontière entre le métal fondu et le métal non fondu.

Zone affectée thermiquement (ZAT) : métal de base qui a subi des modifications de structure sous l'effet de la chaleur, mais qui n'a pas été porté à fusion. Métal de base : zone qui n'a pas été affectée par l'opération de soudage.

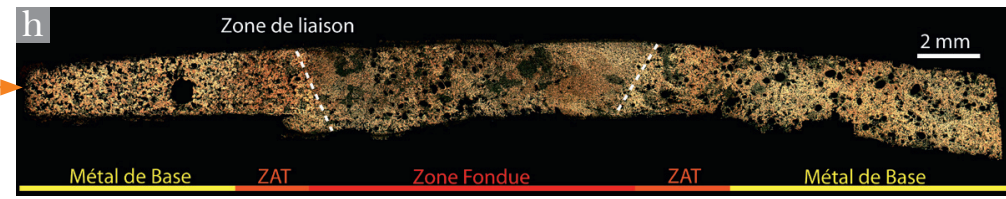

Examen par ultrasons

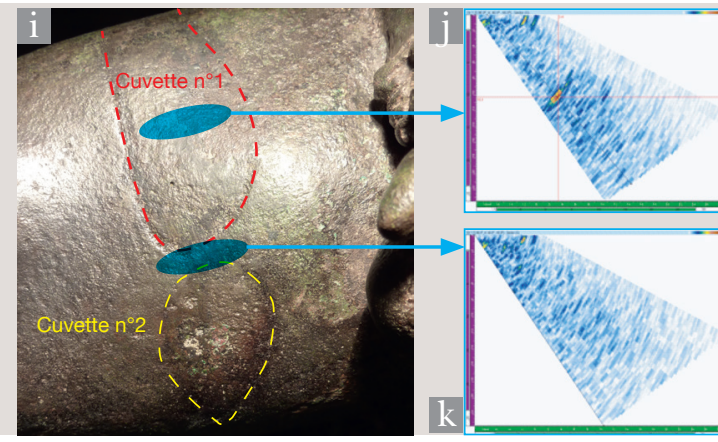

Écho renvoyé à 10 mm d'épaisseur

Aucun écho

Fig. 1. Tableau illustrant diverses techniques d'analyses employées pour l'étude des assemblages soudés de la grande statuaire antique. (c) C2RMF/Institut de Soudure.

De gauche à droite : Fig. 1a $\odot$ A. Azéma. Fig. 1b @ B. Mille. Fig. 1c @ D. Bagault. Fig. 1d @ T. Borel. Fig. 1e @ A. Azéma. Fig. 1f $\odot$ D. Bagault. Fig. $1 \mathrm{~g} \odot$ T. Borel et A. Azéma. Fig. 1h @ A. Azéma. Fig. 1i @ A. Azéma et F. Angelini (Institut de Soudure). Fig. 1j $\odot$ Institut de Soudure. Fig. 1k @ Institut de Soudure. Dimensions des statues : Fig. 1a et 1i : Eros Epiais-Rhus, L. 63 cm. Fig. 1b : Gaulois captif d’Arles, H. 63,5 cm. Fig. 1c, d, e : Apollon d'Évreux, H. 69 cm. Fig. 1f, g : Fragment du dépôt de grands bronzes d'Évreux, L. 14 cm. 
dans la production des grands bronzes grecs puis romains : elle apparaît en effet dès la première moitié du $\mathrm{V}^{\mathrm{e}}$ siècle avant J.-C, et s'est avérée tellement performante qu'elle sera utilisée jusqu'à la fin de la période romaine ${ }^{12}$. L'un des objectifs de cette recherche sur les techniques de soudage est de mettre en évidence l'évolution fine de l'utilisation du procédé « en cuvettes »; les résultats obtenus sur ce point particulier sont pour la première fois présentés ici.

Dans cet article, nous donnerons tout d'abord l'état d'avancement de nos recherches dédiées à l'étude technologique et à la modélisation expérimentale des assemblages soudés. Nous nous attacherons ensuite à présenter une méthodologie d'étude des soudures et ses développements les plus récents, en particulier grâce aux dernières innovations en contrôle non destructif, qui sont détournées de leur vocation industrielle pour de nouvelles applications patrimoniales.

\section{Approches mises en ouvre au C2RMF : quelles analyses pour quels apports?}

\section{Problématique}

Deux paramètres fondamentaux caractérisent une technique de soudage (ou de brasage) : la température de fusion du métal d'apport (inférieure, égale ou supérieure à celle du métal à assembler), et la nature de la source de chaleur employée. En découle le type de la liaison ainsi créé. Les auteurs qui ont abordé le sujet du soudage de la grande sculpture antique en bronze s'accordent tous sur le même principe de base : l'opération était réalisée en versant du bronze liquide (coulée secondaire) dans un espace aménagé entre les deux pièces à assembler (coulées primaires), afin d'assurer une continuité entre ces dernières ${ }^{13}$. La particularité de ce procédé réside donc dans le fait que le bronze versé joue un double rôle : il est à la fois le métal d'apport et la source de chaleur ${ }^{14}$.

Mais la description donnée ci-dessus reste trop succincte pour considérer que cette définition du soudage antique est complète. D’abord, il convient de préciser la composition du métal d'apport (non systématiquement déterminée dans les études) et le type de liaison créé (la proposition formulée n'est souvent fondée que sur des hypothèses). De plus, les études se cantonnent souvent à la description du résultat (la soudure), et non à la reconstruction d'un procédé (le soudage), ce qui ne permet pas d'appréhender les mécanismes en jeu ni le détail de leur mise en œuvre.

\section{Apport de l'étude technologique des statues}

Des approches complémentaires sont requises pour tenter de répondre aux interrogations soulevées.

L'étude des soudures fait désormais partie intégrante de l'étude technologique d'une statue antique en bronze ; la méthodologie développée au C2RMF combine, à chaque fois que cela est possible, les techniques analytiques suivantes :

- l'observation directe des parois externe et interne (si accès) est la plus simple à réaliser ; elle n'en est pas moins fondamentale car elle permet de repérer des indices des différentes étapes de fabrication des statues ${ }^{15}$. Ainsi, différences de corrosion, porosités, surépaisseur de métal peuvent indiquer la présence d'un assemblage (fig. $1^{16}$ ). À noter qu'une ouverture, même très réduite, permet de glisser un endoscope pour inspecter l'intérieur d'une statue.

- la radiographie, basée sur l'absorption différentielle par l'objet à contrôler de rayonnements ionisants (X, gamma), voire de particules (neutrons), permet de détecter les éventuelles hétérogénéités de la paroi de bronze des œuvres étudiées : sur- ou sous-épaisseur, porosités, inclusions, réparations ${ }^{17}$. La radiographie est la technique souvent la plus efficace pour mettre en évidence une soudure. Cette dernière est localisée grâce aux différences de densité, aux discontinuités des parois ou aux potentiels défauts d'assemblage. La technique vient en complément des informations obtenues par observations visuelles : nombre et tracé des assemblages, ce qui renseigne sur le plan de coulée des sculptures ; forme et dimension des assemblages, ce qui témoigne de l'étape de préparation des pièces avant soudage (fig. 1).

- les techniques de contrôle par ultrasons mises au point dans l'industrie, notamment pour inspecter des soudures sur fontes et aciers (pipelines, cuves, etc.) constituent des méthodes d'analyses prometteuses pour l'étude des soudures antiques. Elles permettent d'accéder à une vision globale de l'ensemble des assemblages dans les trois dimensions, grâce à des instruments portables et n'émettant pas de rayonnements ionisants. Les premiers essais, dont nous rendons compte dans la partie Sondages par ultrasons : exploration d'un nouvel angle d'approche de cet article, ont été réalisés sur deux bronzes antiques, et sont extrêmement encourageants. Cela assure à terme des études plus faciles à mettre en place en environnement de musée (les rayonnements ionisants nécessitent la mise en place de zones d'exclusion parfois très contraignantes).

- l'analyse élémentaire du métal permet de confronter la composition du métal des coulées primaires à celle du métal d'apport, et donc notamment de comparer les propriétés thermiques des deux alliages. Une collecte des données disponibles dans la littérature grâce à la base de données Hephaistos $^{18}$, ajoutée aux résultats obtenus dans le cadre du programme conduit au C2RMF, converge vers ce constat : dans la majorité des cas, le métal d'apport (coulée secondaire) était élaboré de manière à avoir une température de fusion légèrement supérieure à celle du métal des coulées primaires ${ }^{19}$. Cette caractéristique est très importante, puisqu'elle exclut a priori un mécanisme de brasage (assemblage obtenu par diffusion de matière), et nous oriente résolument vers un mécanisme de soudage par fusion.

- l'étude métallographique de la microstructure d'une zone d'assemblage est rarement envisageable, puisqu'elle nécessite un prélèvement conséquent (de l'ordre du 
centimètre). Néanmoins, dans les cas où une telle étude est possible ${ }^{20}$, les observations permettent de mieux caractériser le type de liaison obtenue. La prédominance d'un mécanisme de soudage par fusion a été confirmée : les bords des pièces à joindre (métal de base ou coulée primaire) ont été partiellement portés à l'état liquide, le métal ainsi fondu s'est mêlé au métal d'apport. L'ensemble, en refroidissant, a créé une liaison se caractérisant par une continuité de la matière (fig. 1). Certains exemples obligent néanmoins à nuancer l'établissement systématique d'une liaison via ce mécanisme ${ }^{21}$, mais il est en tout cas désormais certain que les fondeurs antiques cherchaient à obtenir des assemblages par soudage par fusion.

Au terme de nos caractérisations des procédés d'assemblage de la grande statuaire antique en bronze, nous proposons donc la dénomination «soudage par fusion au bronze liquide ${ }^{22}$ ", qui permet de décrire à la fois le mécanisme de création de la liaison ( "soudage par fusion»), ainsi que la source de chaleur impliquée («bronze liquide»), en écho à la dénomination des procédés modernes telle que «soudage par fusion à l'arc électrique ».

\section{Apport d'une modélisation expérimentale}

Une définition du procédé de soudage antique est désormais donnée. Mais la question de la mise en œuvre demeure ouverte, en particulier en ce qui concerne le mode d'alimentation en métal d'apport et le contrôle du transfert de la chaleur. Plusieurs chercheurs ont réalisé des expérimentations en appliquant directement le principe de base décrit ci-dessus. Seulement, ces essais ont en majorité été réalisés « grandeur nature » avec pour objectif la reconstruction du procédé de fabrication d'une statue antique ${ }^{23}$. Ceci non seulement implique une mise en ouvre longue et complexe, mais la plupart du temps élude l'étude des mécanismes mêmes soustendant le soudage. Ainsi, le résultat principal de ces tests est qu'il est extrêmement difficile de réussir à souder par fusion toute la longueur du joint en une seule fois. Nous avons adopté une approche différente, en expérimentant le soudage par fusion au bronze liquide à échelle réduite, dans des conditions plus modélisantes. Cela rend alors possible la multiplication des essais, facilite la production de données plus fiables, et donne davantage de liberté dans le choix des paramètres à faire varier.

De tels essais ont été mis en œuvre grâce à la plateforme « haute température » aménagée au C2RMF ${ }^{24}$. Plus de 70 coulées expérimentales ont été effectuées. Chaque pièce à joindre a été modélisée par une éprouvette qui prend la forme d'une plaque de bronze de 50 x $25 \mathrm{~mm}$ et de $4 \mathrm{~mm}$ d'épaisseur. Un essai consiste à positionner deux éprouvettes côte à côte de manière à former un canal entre elles. L'ensemble est maintenu en place grâce à un montage adapté. Du métal en fusion est versé depuis l'une des extrémités du canal ; il s'écoule vers l'autre extrémité, puis tombe dans un bac de rétention. Après quelques secondes de circulation du métal liquide, lorsque l'opérateur estime que le transfert thermique est suffisant pour avoir porté les bords des pièces à la fusion, un bouchon est placé en sortie, ce qui permet au métal d'apport de complètement remplir le canal. La coulée est alors stoppée, l'ensemble refroidit et la zone soudée se solidifie. Composition de l'alliage, forme du canal, température de coulée du métal d'apport, température de préchauffage du métal de base, application ou non d'un flux sont autant de paramètres qui ont été testés d'une coulée à l'autre (fig. 2) ${ }^{25}$.

Les modélisations expérimentales que nous avons conduites ont pour la première fois permis d'expliciter une des principales difficultés à laquelle les fondeurs antiques ont été confrontés. Il s'avère en effet qu'il existe un fort gradient de température entre les deux extrémités du joint, qui fait que la solidification du métal d'apport (et donc la création éventuelle de la liaison) s'effectue dans le sens inverse de sa circulation : le métal se fige d'abord au plus loin de la zone d'entrée où, de fait, les échanges de chaleur avec le métal à souder sont moindres. Notons que ce n'est pas le cas des procédés modernes de soudage, où la source de chaleur se déplace le long du joint et la solidification du métal d'apport s'effectue donc dans le même sens. La conséquence de ce phénomène est que la soudure au bronze liquide est de moins en moins effective à mesure que l'on s'éloigne de la zone d'entrée du métal d'apport (fig. 3).

Ainsi, il apparaît que le succès d'un assemblage soudé antique reposait principalement sur la maîtrise des gradients de température. Il fallait donc trouver un compromis entre deux effets contradictoires : transférer le plus efficacement possible la chaleur du métal d'apport suivant l'axe du joint - et donc disposer d'un métal d'apport suffisamment chaud, tout en limitant et en homogénéisant la diffusion latérale de la chaleur de façon à ce que les bords du canal ne fondent pas uniquement au niveau de la zone d'alimentation. Parmi les paramètres susceptibles d'influer sur la réussite du soudage, nous avons montré que la mouillabilitée ${ }^{26}$ des surfaces, ainsi que la forme du joint peuvent améliorer significativement le rendement.

Il est facile d'améliorer la mouillabilité des surfaces à joindre. Cela favorise la circulation du métal d'apport dans le canal, tout en ralentissant le transfert de chaleur depuis le métal d'apport vers le métal de base. Il suffit d'isoler temporairement métal de base et métal d'apport par une substance, que nous nommons "flux ». L'éventail des substances ayant pu servir de flux est grand, nous en avons testé quelques-unes (poudre de malachite, poudre d'os, huiles...). Le flux doit posséder un coefficient d'échange de chaleur relativement faible, afin dans un premier temps de ralentir le transfert de chaleur et donc de favoriser le maintien à l'état liquide du métal d'apport pour que celui-ci circule d'un bout à l'autre de l'assemblage, ce qui homogénéise le transfert de chaleur sur la longueur du joint. Dans un deuxième temps, le flux doit se transformer ou disparaître pour que le bord des pièces à joindre, partiellement porté à fusion, et le métal d'apport puissent entrer en contact et se 
Fig. 2. Schéma du principe des essais de soudage par fusion au bronze liquide, réalisés au C2RMF, montrant les plaques à souder (jaune clair) et le métal d'apport (ocre) (a) et schéma en coupe transversale montrant le dispositif expérimental et les deux types de préparation au soudage testés (b). (C) C2RMF/A. Azéma.

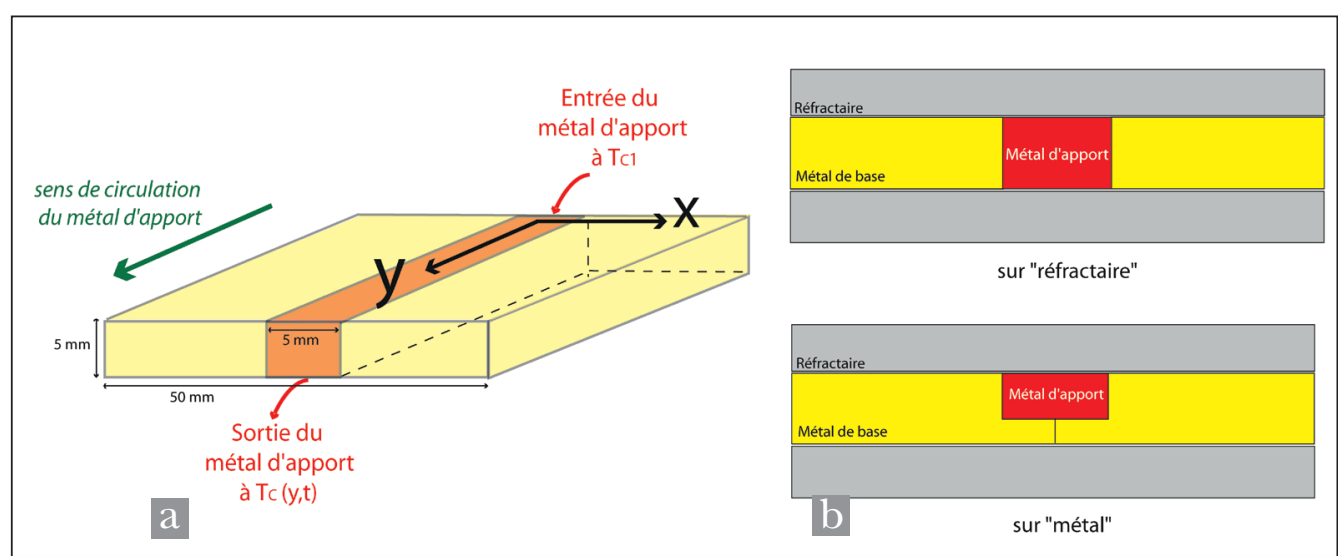

solidifier ensemble. Outre l'intérêt évident du recours à une telle substance, son utilisation a été mise en évidence sur plusieurs coupes métallographiques de soudures antiques ${ }^{27}$.

Le second paramètre est directement lié à la quantité de chaleur fournie. En jouant sur le rapport des surfaces à joindre (bords des pièces) avec le volume du joint rempli par le métal d'apport, il est possible de limiter le gradient thermique sur la longueur du joint. Plus ce rapport est faible, plus il sera facile d'obtenir un joint soudé de grande longueur. La manière d'agir sur ce paramètre est donc liée à la préparation au soudage. Plusieurs aménagements particuliers, observés sur les bronzes antiques, ont vraisemblablement été développés dans ce but :

- la présence d'un fond métallique est déterminante : si le fond du canal est un matériau réfractaire (fig. 2a et 2b, en haut) le gradient longitudinal est fortement diminué, et le gradient latéral fortement augmenté, ce qui est donc très favorable au soudage. En revanche, si le fond du canal est métallique (fig. 2b, en bas), le positionnement des pièces est plus précis et ne nécessite pas la mise en place d'un réfractaire sous la soudure, mais le gradient longitudinal est beaucoup plus difficile à limiter.

- une autre possibilité était d'élargir le joint afin d'y verser davantage de métal d'apport et donc augmenter la quantité de chaleur pouvant être mobilisée. Le système de cuvettes, si fréquemment observé sur les statues antiques, apparaît ainsi comme une manière de créer des "réservoirs » de chaleur répartis tout le long du joint soudé (fig. 4a, et voir plus loin le Gaulois captif d'Arles). On observe même dans certains cas que la soudure prend la forme d'une succession de cuvettes qui se touchent, et donc « ouvertes » sans véritable présence d'un cordon (fig. 4c). Il y a aussi un cas, jusqu'à présent uniquement observé sur des objets de la période romaine et gallo-romaine, et qui pose question : les cuvettes sont non communicantes tant le cordon inter-cuvettes est fin (fig. 4b) ${ }^{28}$. Cela veut-il dire que la soudure a été effectuée cuvette par cuvette?
Fig. 3. Vues de dessus de deux éprouvettes expérimentales soudées par fusion au bronze liquide ; à gauche, radiographie $\mathrm{X}$; à droite, coupe métallographique préparée dans le plan des éprouvettes (attaque chimique au perchlorure de fer). (C) C2RMF/T. Borel et A. Azéma.
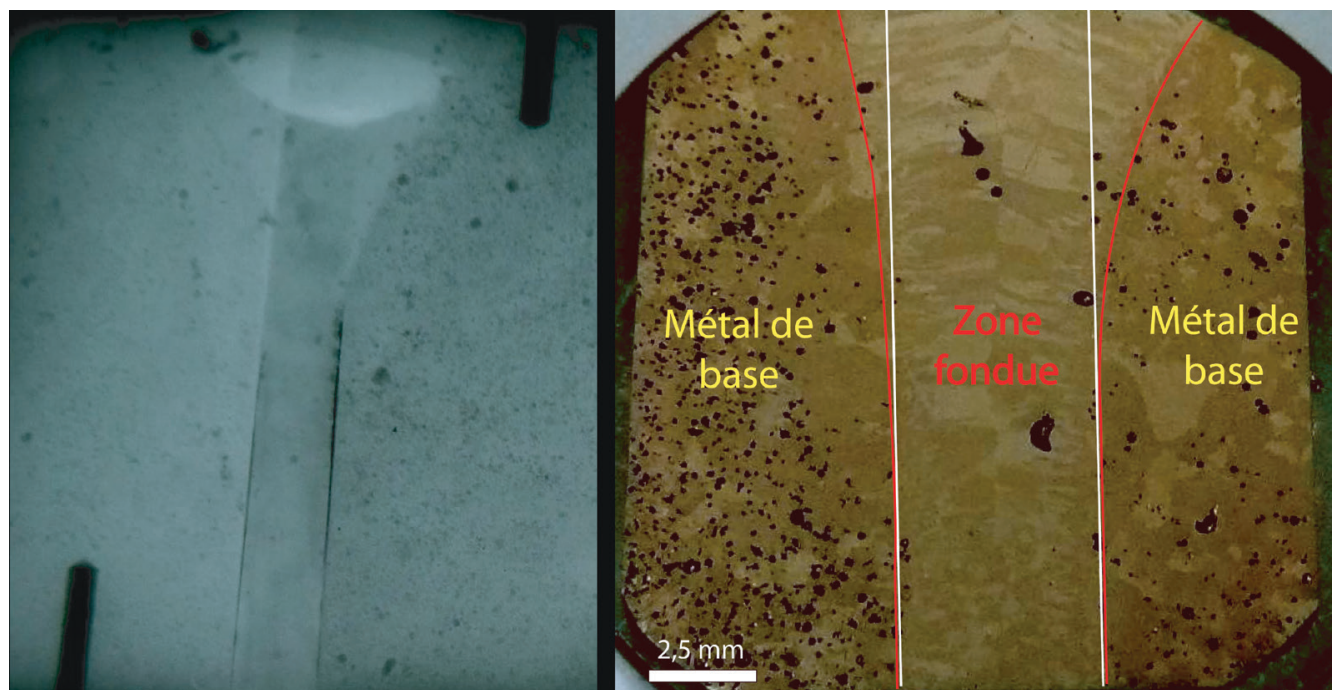


\section{Préparation en cuvettes communicantes \\ métal de base}

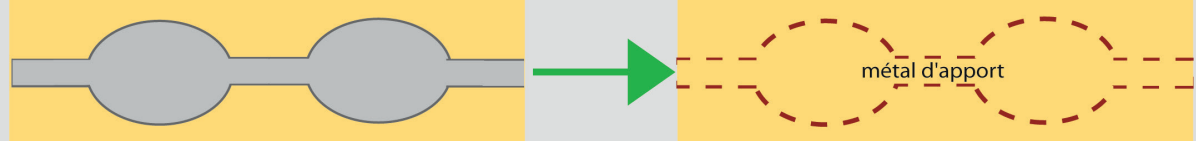

métal de base

a

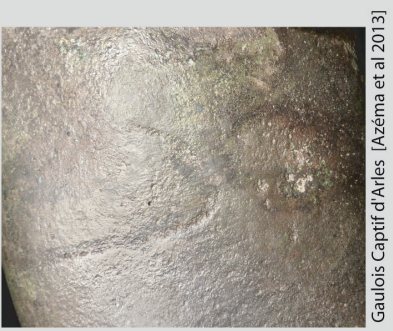

\section{Préparation en cuvettes non communicantes}

métal de base

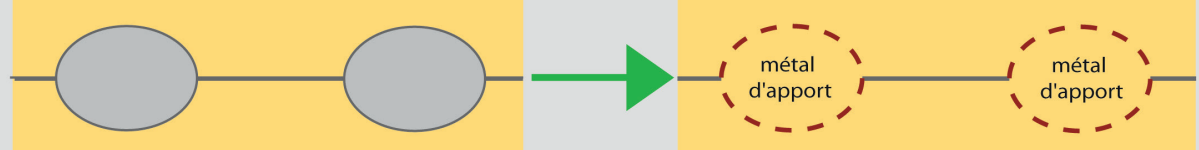

métal de base

b

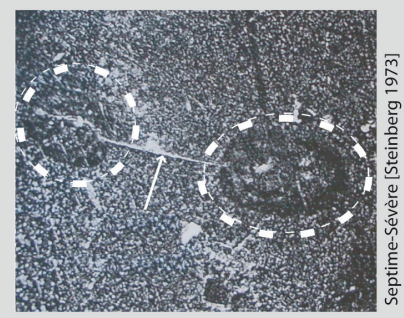

\section{Préparation en cuvettes ouvertes}

métal de base

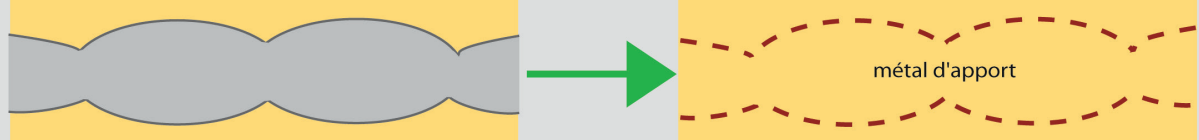

métal de base

Fig. 4. Typologie des préparations au soudage en cuvettes observées à ce jour sur la grande statuaire grecque et romaine en bronze. (c) C2RMF. Schémas : A. Azéma et B. Mille ; Photo D. Bagault [Azéma et al., 2013] ; Photo A. Steinberg [Steinberg, 1973] ; Photo A. Chauvet [Mille et Azéma, 2012].

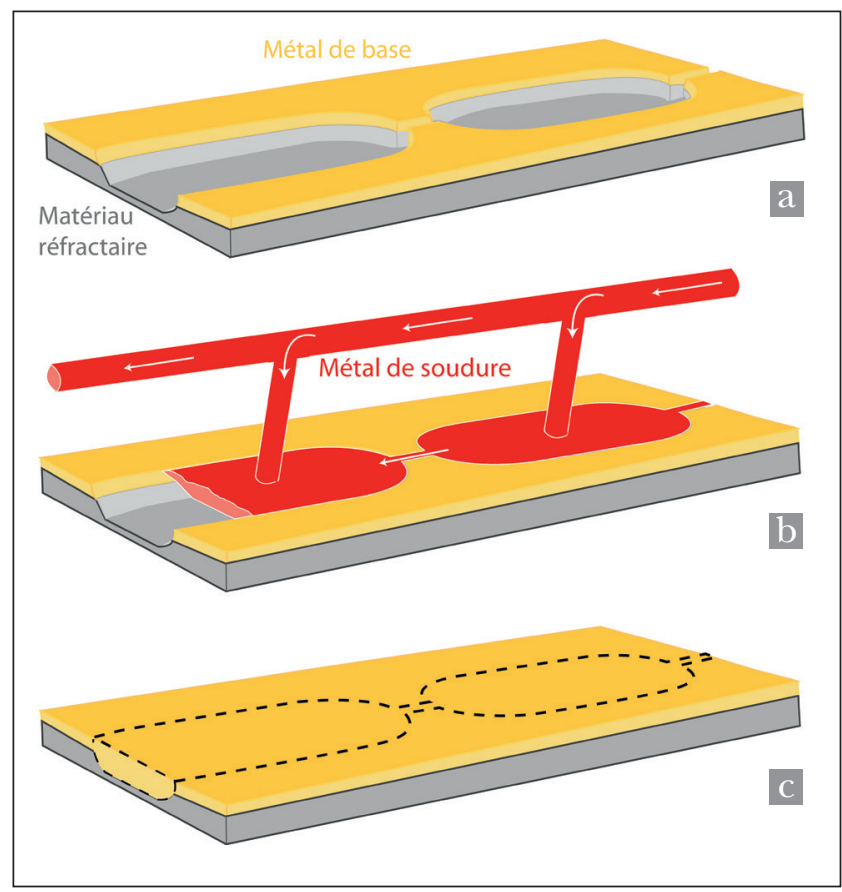

Fig. 5. Schéma illustrant l'hypothèse de la multiplication des alimentations en métal d'apport pour le soudage de cuvettes «non communicantes ». ( C C2RMF/A. Azéma et B. Mille.
- plusieurs indices laissent en fait penser que le soudage en cuvettes non communicantes était, au moins dans certains cas, également effectué en une seule et unique opération de coulée. L'astuce des fondeurs antiques aurait résidé dans la multiplication des entrées du métal d'apport, et donc l'alimentation et la communication des cuvettes par un système externe (fig. 5). Après élimination de ce système d'alimentation, on aboutissait à une soudure dont les cuvettes sont non communicantes via la paroi métallique (fig. 4b).

Ainsi, la modélisation expérimentale du procédé de soudage par fusion au bronze liquide, en plus de pointer les difficultés surmontées par les fondeurs antiques, nous conduit à mieux comprendre les aménagements particuliers que nous observons sur les bronzes antiques. Reste maintenant à vérifier l'influence des paramètres-clés cités ci-dessus par la modélisation de joints de plus grande longueur sur des surfaces incurvées, voire même de se lancer dans une véritable reconstruction expérimentale, ce qui nécessitera alors la collaboration d'une fonderie d'art. 


\section{Sondages par ultrasons : exploration d'un nouvel angle d'approche}

Quel que soit l'angle choisi pour la simulation expérimentale, l'étude directe des témoins matériels conservés sur les statues antiques reste primordiale. À cet effet, nous avons cherché à développer le panel d'outils pour révéler des indices de leur fabrication. Si radiographies et tomographies sont à ce jour les méthodes les plus satisfaisantes (voir ci-dessus), elles nécessitent le déplacement de l'objet au laboratoire ou le recours à du matériel portable dont la mise en ouvre peutêtre très contraignante dans l'environnement muséal. Pour ce qui concerne les coupes métallographiques, elles ne sont pas seulement rarement envisageables; leur observation ne permet pas de généraliser à l'ensemble des assemblages présents sur une statue (variation de la morphologie du joint suivant sa localisation sur la statue, fréquences des défauts). Ainsi, la mise en ouvre d'analyses par ultrasons apparaît comme une approche très prometteuse de l'étude des techniques antiques de soudage, étant à la fois globale et locale, pouvant fournir des données dans les trois dimensions de l'espace, restant complètement non invasive, et s'effectuant sans émission de rayonnements ionisants.

\section{Une première application aux grands bronzes antiques}

Principe du contrôle ultrasonore multi-éléments

Cette technique de CND (Contrôle Non Destructif) dérive de l'échographie médicale. Elle permet l'inspection de grandes surfaces ou longueurs soudées. Contrairement aux ultrasons classiques qui utilisent des transducteurs monoélément, la technique ultrasonore multi-éléments (PAUT : Phased-Array Ultrasonic Testing) est basée sur l'exploitation

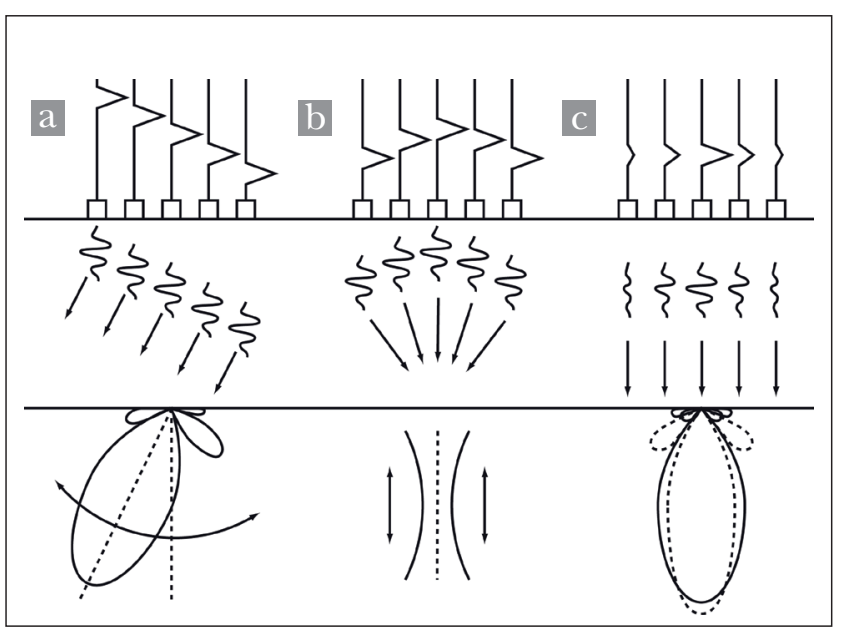

Fig. 6. Fonctions fondamentales de pilotage d'un traducteur multi-éléments : (a) déflexion électronique (base du S-scan), (b) focalisation électronique, (c) mesure par atténuation des lobes latéraux. (C) Institut de Soudure.
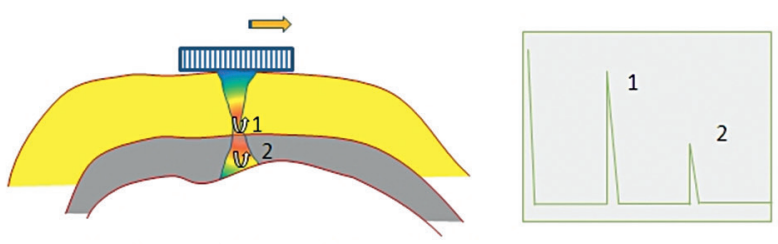

- Le faisceau est formé et déplacé électroniquement. - Génération d'un écho sur la paroi de bronze (1).

Note : \# Les amplitudes des échos 1 et 2 dépendent de l'orientation de la paroi par rapport au faisceau et de l'impédance acoustique. de temps à partir de laquelle la mesure d'épaisseur est déduite est effectuée entre l'écho 1 et sa première répétition.

Fig. 7. Principe de base du contrôle ultrasonore schématiquement appliqué ici sur une paroi courbe de bronze (en jaune) qui serait en contact avec un autre matériau, comme par exemple un noyau réfractaire (en gris). (c) Institut de Soudure.

d'éléments piézo-électriques de petites dimensions agencés en réseaux linéaires ou matriciels, de formes variées. Les éléments sont excités individuellement ou par groupe par une impulsion brève, envoyée à différents intervalles de temps, afin de générer dans le matériau à contrôler un train d'onde caractérisé par sa vitesse et sa direction de propagation, ainsi que sa répartition énergétique le long du parcours ultrasonore. Les électroniques permettant de piloter un traducteur multiéléments sont en général dotées des fonctions fondamentales de contrôle de faisceau suivantes (fig. 6) : le balayage électronique, le balayage sectoriel, la déflexion électronique (a), la focalisation, laquelle peut être dynamique (b) et, plus rarement, l'atténuation des lobes latéraux (c).

La figure 7 rappelle le principe de base utilisé lors du contrôle ultrasonore d'une paroi et indique les échos susceptibles d'être pris en compte.

\section{Exemple du Gaulois captif d'Arles}

Nous rendons compte ici des principaux résultats d'une étude publiée récemment ${ }^{29}$. Les zones de soudure de la statue du Gaulois captif ${ }^{30}$ sont pour la plupart visibles à l'œil nu. Ainsi, l'objectif des examens par ultrasons n'était pas de les localiser, mais plutôt d'en donner une caractérisation morphologique en trois dimensions par combinaison de plusieurs types de balayages (linéaire et sectoriel). Plusieurs assemblages ont ainsi été sondés dont la soudure de la jambe droite, et celle du bras gauche. 


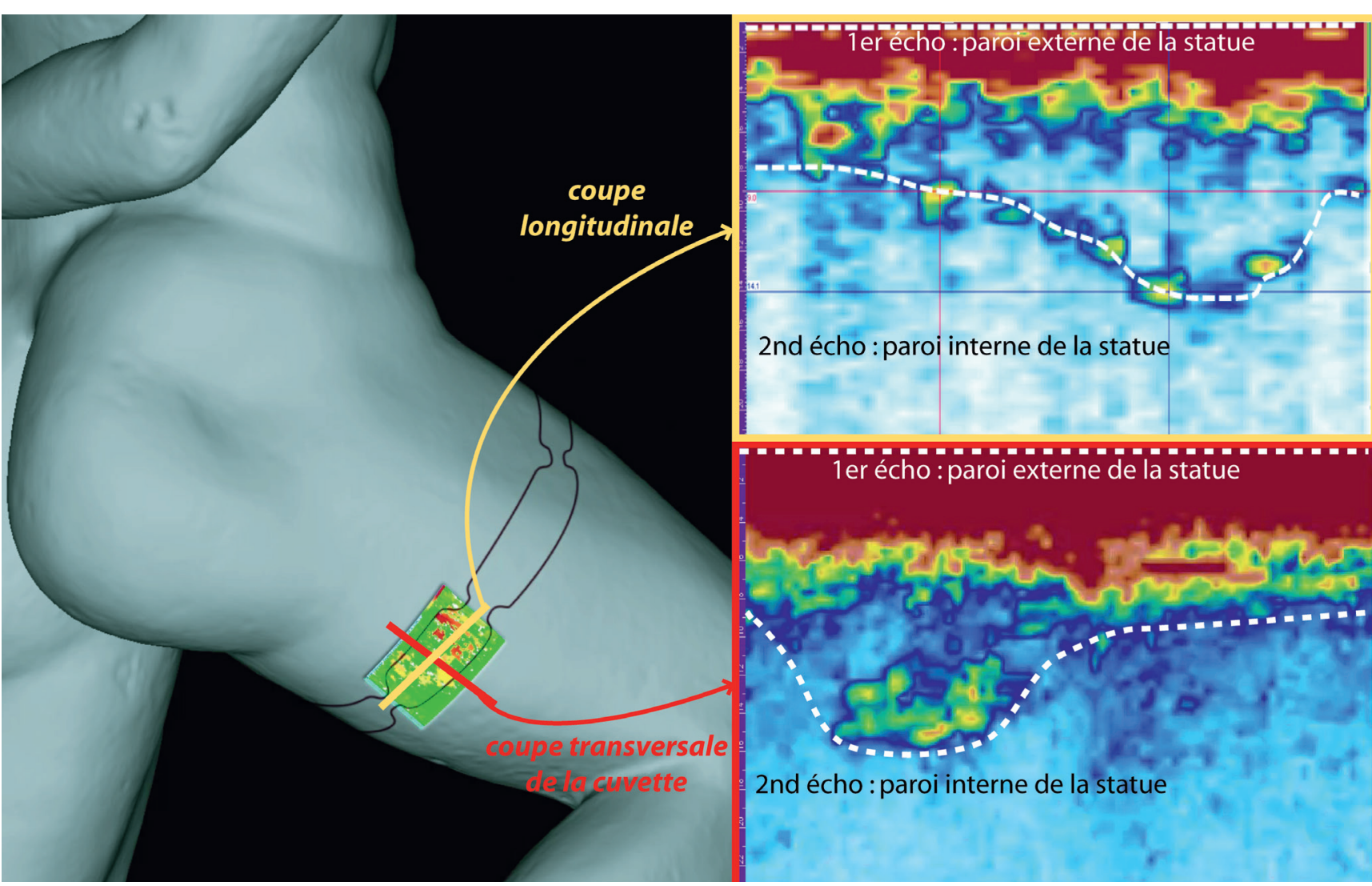

Fig. 8. Profilométrie ultrasonore d'une cuvette du Gaulois captif d'Arles (coupe longitudinale en haut à droite, coupe transversale en bas à droite). (C) Institut de Soudure/C2RMF.

À gauche :@ Institut de Soudure/D. Kolin, G. Blaise, A. Azéma. À droite : @ Institut de Soudure.

Un premier examen a été réalisé dans la zone d'une cuvette de soudure située à l'arrière de la cuisse droite. Il en a résulté un C-scan temporel (fig. 8, à gauche) correspondant à une vue 2D plane (vue de dessus déroulée) représentant les variations d'épaisseur de la surface examinée (du vert au rouge, l'épaisseur varie de $6 \mathrm{~mm}$ à $14 \mathrm{~mm}$ ). Cet examen a permis d'extraire des coupes transversales de cette zone et ainsi de visualiser avec précision le profil de la soudure (fig. 8, à droite), fournissant une morphologie précise dans les trois dimensions.

Dans le cadre d'un second examen, au niveau de la zone soudée à l'avant de la cuisse droite, des balayages sectoriels ont été effectués ; les résultats obtenus permettent de conclure que les cuvettes étaient vraisemblablement communicantes (fig. 4a).

Grâce aux examens réalisés, la préparation au soudage mise en œuvre par les fondeurs antiques sur la statue du Captif a été précisément décrite : les deux pièces à assembler étaient positionnées côte à côte tout en maintenant un espace vacant entre elles, ponctué de cuvettes destinées à recevoir le métal de soudure. Un système constitué de matériaux réfractaires a nécessairement été mis en place pour éviter les fuites de métal à l'intérieur de la statue. Si l'on compare ce procédé au soudage moderne, cette technique est assimilable au soudage sur latte ${ }^{31}$, la latte ayant pour fonction de canaliser le bain de soudure et en limiter la dissipation thermique.

\section{Exemple de l'Apollon de Piombino}

Quatre zones de l'Apollon de Piombino ${ }^{32}$ ont été sondées, préférentiellement au niveau des soudures en cuvettes identifiées lors des analyses et examens préalables ${ }^{33}$. Un exemple de cartographie ultrasonore réalisée en mode temporel (sans utilisation d'encodeur de déplacement mécanique) est présenté en figure 9. La cartographie a été réalisée sur une longueur de $100 \mathrm{~mm}$ au droit d'une zone présentant visuellement une soudure en cuvettes sur la face externe du bras droit (fig. 9).

Des coupes de type S-scan, représentant une vue de coupe issue du balayage angulaire du faisceau ultrasonore, présentées sur la figure 9, traduisent les variations de l'épaisseur du bras droit. Comme dans le cas du Gaulois captif, l'épaisseur de la cuvette s'est révélée plus importante que celle des pièces qu'elle assemble. En outre, il apparaît que la zone supposée de soudure en cuvettes présente une plus grande absorption aux ultrasons que la zone correspondant au métal de base, ce qui indique des structures métallurgiques différentes. Au-delà de la caractérisation des soudures, cette technique 


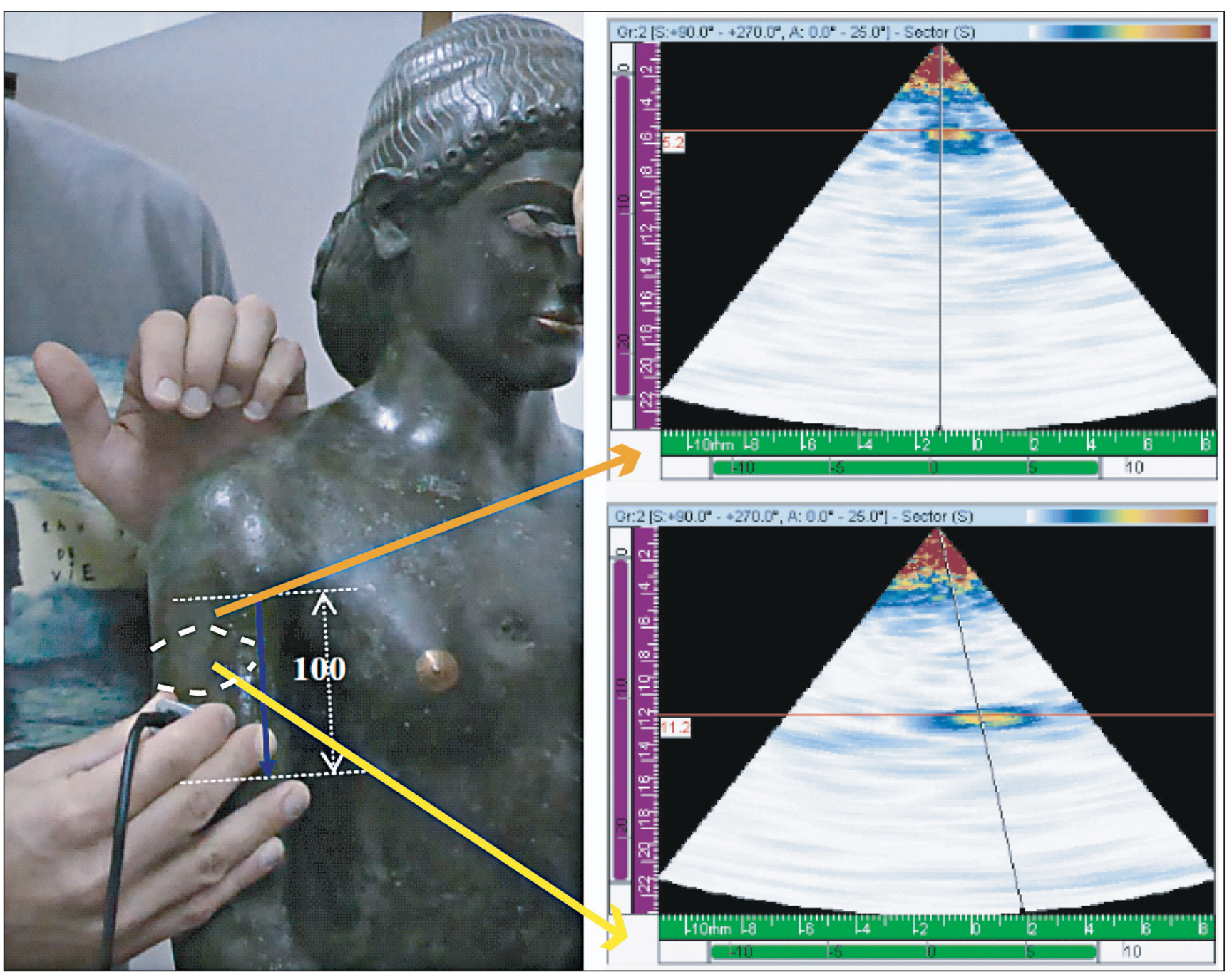

Fig. 9. Repérage de la zone contrôlée depuis la face externe du bras droit de l’Apollon de Piombino. (C) Institut de Soudure.

de cartographie ultrasonore a démontré son grand potentiel pour la réalisation d'une profilométrie des parois internes et la détection des défauts ${ }^{34}$.

\section{Développement en cours et perspectives}

Comme illustré dans les paragraphes précédents, la plupart des systèmes d'imagerie ultrasonore actuellement commercialisés ne délivrent pas l'image vraie du défaut, mais une représentation complexe qui nécessite une interprétation demandant une grande expérience.

C'est pourquoi l'Institut de Soudure a entrepris, avec le support de la BPI (Banque Publique d'Investissement), une étude ayant pour objet d'étudier et de réaliser un démonstrateur de système de tomographie ultrasonore capable de reconstruire de manière réaliste des indications ultrasonores, y compris sur des pièces de géométrie complexe comme c'est le cas pour la grande statuaire antique.

Ce système innovant exploite les dernières avancées technologiques d'algorithmes de reconstruction d'image. Ces algorithmes sont basés sur un principe de focalisation ultrasonore et d'inversion des données, dit TFM (Total Focusing Method)/FMC (Full Matrix Capture). D'une certaine manière, cela revient à obtenir une image où l'on focalise l'énergie ultrasonore en tout point.

Néanmoins, les signaux délivrés ne sont pas associés spatialement à l'objet examiné et ne donnent pas de représentation 3D de la zone d'examen. Le développement d'algorithmes de reconstruction 3D a donc été nécessaire. Un exemple de reconstruction obtenue avec le démonstrateur, effectuée par l'Institut de Soudure sur la soudure arasée d'un tube en acier de diamètre et d'épaisseur connus, est présenté en figure 10, ainsi qu'une coupe réalisée au droit du trait 


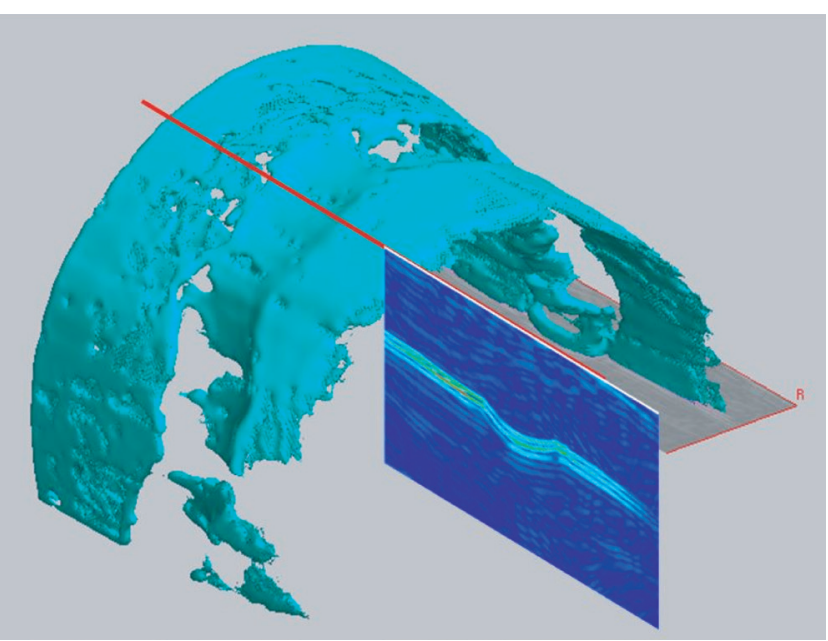

Fig. 10. Tomographie ultrasonore d'une soudure.

(c) Institut de Soudure.

rouge. On comprend aisément tout l'intérêt d'un tel système pour examiner aussi les soudures des bronzes antiques.

Reste la difficulté imposée par la géométrie des statues. Les sondes ultrasonores classiques sont rigides et les sabots sur lesquels elles sont fixées ont un rayon de courbure fixe, ne permettant pas une transmission optimale des ultrasons. Dans ce domaine, de nouveaux développements sont prometteurs. En effet, il existe aujourd'hui des sondes ultrasonores conformables qui permettraient d'assurer un couplage optimal dans presque toutes les parties d'une statue.

\section{Conclusion}

Dans le cadre du programme de recherche sur l'évolution des techniques de la grande statuaire antique en bronze, conduit par le C2RMF et le musée du Louvre, nous avons mis un accent particulier sur les techniques de soudage, et ces travaux ont fortement contribué à une meilleure compréhension des mécanismes impliqués. Un éventail de techniques d'analyse a été mis en œuvre et d'autres sont en développement pour tenir compte des limites déontologiques imposées par les objets du patrimoine, telles que la difficulté de prélever des échantillons de taille centimétrique pour la réalisation de coupes métallographiques. La complémentarité de ces techniques a permis de reconstruire progressivement la chaîne opératoire de ce procédé et d'identifier des variantes qui caractérisent des pratiques d'atelier différentes. Cela montre aussi que les techniques d'assemblage portent en germe un fort potentiel pour la discrimination selon des critères d'ordre géographiques ou chronologiques. Cependant, il est encore trop tôt pour se lancer dans une reconstitution de l'histoire du soudage, car le corpus de sculptures dont les assemblages ont été étudiés de manière approfondie demeure très insuffisant. Pour encourager la production de telles données, il conviendrait d'inciter à l'examen systématique des assemblages des sculptures selon une méthodologie spécifique. Une telle méthodologie sera d'autant plus opérante que sa mise en œuvre sera facile, et qu'elle permettra d'accéder à plusieurs niveaux de caractérisation des soudures. À ce titre, les techniques d'analyses dont les résultats peuvent faire l'objet de reconstruction 3D sont les plus prometteuses, qu'il s'agisse de tomographie $\mathrm{X}$ (technique appliquée en routine dans l'industrie, mais qui reste souvent inaccessible pour les objets du patrimoine), ou bien des derniers développements d'examens par ultrasons qui offrent d'intéressantes perspectives vers la réalisation de véritables tomographies ultrasonores à l'aide d'instruments portables sur le lieu même de conservation des ouvres.

\section{Notes}

1. La meilleure description des soudures antiques est longtemps restée celle donnée par François Rever dans son étude de l'Apollon de Lillebonne ; Rever, 1824, p. 14-15. Pour une présentation générale de cette statue, voir Robcis et al., dans ce volume.

2. Synthèse des débats du $\mathrm{XX}^{\mathrm{e}}$ siècle sur les procédés de fonte dans Mattusch, 1988, p. 22-30.

3. Craddock, 1977, p. 103-123.

4. Steinberg, 1973, p. 103-138.

5. Bronzes de Riace, vers 460 av. J.-C. : statue A, inv. 12801, H. $198 \mathrm{~cm}$ et statue B, inv. 12802, H. 197 cm, Reggio di Calabria,
Museo archeologico nazionale. Formigli, 1984 , p. $107-142$.

6. Janietz Schwarz, Rouiller, 1996 ; Janietz Schwarz, 2000.

7. Mattusch, 1996.

8. II $^{\mathrm{e}}$ siècle ap. J.-C., inv. A6286, Orléans, musée historique et archéologique, H. 105 cm, Mille, 2007, p. 264-265 ; Azéma, Mille, 2010, p. 20-22.

9. Mille, Robcis, 2012, p. 101-116.

10. Mille et al., 2012

11. Karnis, Mille, 2015, p. 97-105 ; voir Descamps-Lequime et Mille, dans ce volume ; Mille, Descamps-Lequime, à paraître. 12. Formigli, 1984, p. 107-142 ; Mille, 2007.

13. Steinberg, 1973, p. 103-138.
14. Azéma, 2013, p. 142-189.

15. Mille, Serneels, 2012, p. 104-107.

16. Azéma et al., 2012, p. 153-172 ; Mille, Robcis, 2009, p. 162-165.

17. Borel, 1995.

18. Voir Descamps-Lequime et Mille, dans ce volume.

19. Azéma, 2013, tableau 2.4 et p. 236239.

20. Azéma, 2013, fig. 2.42.

21. Mille, 2007, p. 88-99 et p. 264-265 ; Mille, Azéma, 2012, p. 24-33.

22. Azéma et al., 2010, p. 20-22 ; Azéma et al., 2011, p. 71-80 ; Azéma, 2013, chap. 2, p. 103-189.

23. Peltz, 2010 ; Zimmer et al., 2011, p. 264-273 ; Peltz, Schalles, 2011. 
24. Bourgarit, Thomas, 2011, p 8-16. 25. Azéma et al., 2011, p. 71-80.

26. La mouillabilité constitue la faculté pour une substance solide de recevoir un liquide en lui permettant de s'étaler sur la surface la plus grande possible ; Quéré, 2003.

27. Caumont et al., 2006, p. 173-195 ;

Darblade-Audoin, Mille, 2008, p. 31-68.

28. Mille, 2007, p. 88-99 et p. 264-265.

29. Azéma et al., 2013, p. 477-486.

30. Gaulois captif d'Arles, $\mathrm{I}^{\mathrm{er}}$ siècle av. J.-C., inv. Rho.2007.06.1963, H. 69 cm, Musée Départemental Arles Antique (MDAA).

31. Murry, 1994, p. M715-3.

32. Apollon de Piombino, dernier quart du $\mathrm{II}^{\mathrm{e}}$ siècle av. J.-C ., inv. Br 2, H. $115 \mathrm{~cm}$, musée du Louvre, département des Antiquités grecques, étrusques et romaines Sur cette œuvre, voir également l'article de Nathan Badoud dans ce volume.

33. Mille, Descamps-Lequime, à paraître. 34. Angelini, 2014.

\section{Bibliographie}

Azéma A., Mille B., 2010, « La grande sculpture antique en bronze assemblée par soudage par fusion ", Soudage et techniques connexes, mars-avril, Institut de Soudure, p. 20-22.

Azéma A., Mille B., Echegut P., De Sousa Meneses D., 2011, "An experimental study of the welding techniques used on large Greek and Roman bronze statues", Historical Metallurgy Society, 45 (2), p. 71-80.

Azéma A., Mille B., Pilon F., Birolleau J.-C., Guyard L., 2012, «Étude archéométallurgique du dépôt de grands bronzes du sanctuaire galloromain du Vieil-Évreux (Eure)", ArchéoSciences, revue d'Archéometrie n ${ }^{\circ} 36$, p. 153-172.

Azéma A., Angelini F., Mille B., Framezelle G., Chauveau D., 2013, "Ultrasonic phased array contribution to the knowledge of the fusion welding process used on roman large bronze statues", Welding in the World, 57(4), p. $477-486$

Azéma A., 2013, « Les techniques de soudage de la grande statuaire antique en bronze : étude des paramètres thermiques et chimiques contrôlant le soudage par fusion au bronze liquide ", Thèse de doctorat de l'université Pierre-et-Marie-Curie (Paris VI) spécialité Chimie physique et chimie analytique de Paris Centre (ED 388), sous la direction de D. Bourgarit et l'encadrement de B. Mille, 251 p., https//tel.archives-ouvertes.fr/tel00918829/, consulté le $1^{\text {er }}$ mai 2017.

Borel T., 1995, « La radiographie des objets d'art », Technè, 2, p. 146-157.

Bourgarit D., Thomas, N., 2011, "The ancient brass cementation processes: from laboratory to field experiments", Historical metallurgy, 20 (1), p 8-16.

Caumont O., Margarit X., Mille B., Piccardo
P., Rolley C., 2006, « Un bras d'un empereur romain en bronze à Essegney (Vosges) ", Revue archéologique de l'Est, tome 55, p 173-195.

Craddock P., 1977, "The composition of the copper alloys used by the Greek, Etruscan and Roman civilizations. 2. The Archaic, Classical and Hellenistic Greeks", Journal of Archaeological Sciences, 4(2), p. 103-123.

Darblade-Audoin M.-P., Mille B., 2008, «Le pied de bronze colossal de Clermont-Ferrand ", Monuments et mémoires de la fondation Eugène-Piot, tome 87, Académie des Inscriptions et Belles-Lettres, Paris, p. 31-68.

Formigli E., 1984, « La tecnica di costruzione delle statue di Riace », Due Bronzi da Riace, Bolletino d'Arte, Serie speciale 3, p. 107-142.

Janietz Schwarz B., Rouiller D., 1996 Ein Depot zerschlagener Grossbronzen aus Augusta Raurica: die Rekonstruktion der beiden Pferdestatuen und Untersuchungen zur Herstellungstechnik, Augst,

Römermuseum (Forschungen in Augst, 20), $238 \mathrm{p}$

Janietz Schwarz B., 2000, Ein Depot zerschlagener Grossbronzen aus Augusta Raurica: die Rekonstruktion der Gewandfiguren, Augst, Römermuseum, (Forschungen in Augst, 30), 208 p.

Karniš Vidovič I., Mille B., 2015, “The Croatian Apoxyomenos - New Insights into its Manufacturing Technique", in Deschler-Erb, E., Della Casa, P. (dir.), New Research on Ancient Bronzes, acta of the XVIIIth International Congress on Ancient Bronzes, Zurich Studies in Archaeology, Zurich: Chronos Verlag, p. $97-105$.

Mattusch C. C., 1988, Greek Bronze Statuary: From the Beginnings Through the Fifth Century B.C., Ithaca \& London: Cornell University Press, $256 \mathrm{p}$.

Mattusch C. C., 1996, The fire of Hephaïstos: Large Classical Bronzes from North American Collections, Cambridge, MA: Harvard University Art Museum, 359 p.

Mille B., 2007, «Étude technique du cheval de bronze de Neuvy-en-Sullias ", Le cheval et la danseuse, à la redécouverte du trésor de Neuvy-en-Sullias, musée des Beaux-Arts d'Orléans (Paris : éditions Somogy, 13 mars-26 août 2007), p. 88-99 et p. 264-265.

Mille B., Azéma A., 2012, « Nouvelles données de laboratoire sur l'Éphèbe d'Agde ", dans Berard-Azzouz, $\mathrm{O}$ (dir.), De l'Éphèbe à l'Alexandre d'Agde, musée de l'Éphèbe, Agde, p. 24-33.

Mille B., Rossetti L., Rolley C., Bourgarit D., Formigli E., Pernot M., 2012, «Les deux statues d'enfant en bronze (Cap d'Agde) : étude iconographique et technique », dans Denoyelle M., Descamps-Lequime S., Mille B. et Verger S. (dir.), Actes du Colloque "Bronzes grecs et romains, recherches récentes ", Hommage à Claude Rolley du 16-17 juin 2009, INHA, http://inha.revues.org/3949, consulté le $1^{\mathrm{er}}$ mai 2017.
Mille B., Descamps-Lequime S., à paraître "A Technological Re-Examination of the Piombino Apollo", in Lapatin, K., Daehner, J. (eds.), 19th International Congress on Ancient Bronzes, 13-17 October 2015, Los Angeles, Getty Trust.

Mille B., Robcis. D, 2009, " Note sur la technique de fabrication du captif ", dans Picard P., Long L. (dir.), César, le Rhône pour mémoire, vingt ans de fouilles dans le fleuve à Arles, MDAA, 24 octobre 2009-19 septembre 2010, Actes Sud, Arles, p. 162-165.

Mille B., Robcis D., 2012, «Le cas des grands bronzes antiques. Étudier pour restaurer ou restaurer pour étudier?", $L$ a restauration des peintures et des sculptures : connaissance et reconnaissance de l'œuvre, éditions Armand Colin, p. 101-116.

Mille B., Serneels V., 2012, «Pas de bras, pas de... L'envers du décor à Arconciel ", Archeoquiz, enquêtes archéologiques fribourgeoises, Fribourg, p. 104-107.

Murry G., 1994, «Soudage et soudabilité métallurgique des métaux ", Techniques de l'ingénieur, vol. MC2, n ${ }^{\circ} 715$, p. M715-1 à M715-34.

Peltz U., 2010, «Die Antike Technik des Angusschweissen ", [En ligne], consulté le 12 décembre 2012. URL : http:// www.uwepeltz.de/

Peltz, U., Schalles H.-J., 2011, Der Xantener Knabe. Technologie, Ikonographie, Funktion und Datierung, Philipp von Zabern Verlag, 188 p.

Quéré D., 2003, « Lois du mouillage et de l'imprégnation ", Techniques de l'Ingénieur, vol. J2, n J2140, p 1-15.

Rever F., 1824, Description de la statue fruste, en bronze doré, trouvée à Lillebonne, arrondissement du Havre, département de la Seine-Inférieure; suivie de l'analyse du métal, avec le dessin de la statue, et les tracés de quelques particularités de cette Antique, seconde édition, Évreux, $45 \mathrm{p}$.

Steinberg A., 1973, "Joining Methods on Large Bronze Statues: some experiments in ancient technology", in William J-Young (dir.), Application of Science in examination of works of art, Actes de la conférence (Boston, 15-19 juin 1970), Museum of Fine Arts, p. 103-138.

Zimmer G., Wirth M., Ellerbrok R., BührigPolaczek A., 2011, « Stückungstechnik griechischer Bronzestatuen-Kunst und technologisher Fortschritt ", Giesserei $98, \mathrm{n}^{\circ}$ 6, p. 264-273.

Document inédit

Angelini F., 2014, Étude de la profilométrie interne de l'Apollon de Piombino par ultrasons phased array, Rapport technique de l'Institut de Soudure, PA-01, 11 p. 